\title{
Optical factors determined by the T-matrix method in turbidity measurement of absolute coagulation rate constants
}

\author{
Shenghua Xu, Jie Liu, Zhiwei Sun* \\ NML, Institute of Mechanics, Chinese Academy of Sciences, Beijing 100080, People's Republic of China
}

Received 2 June 2006; accepted 11 August 2006

Available online 17 August 2006

\begin{abstract}
Turbidity measurement for the absolute coagulation rate constants of suspensions has been extensively adopted because of its simplicity and easy implementation. A key factor in deriving the rate constant from experimental data is how to theoretically evaluate the so-called optical factor involved in calculating the extinction cross section of doublets formed during aggregation. In a previous paper, we have shown that compared with other theoretical approaches, the T-matrix method provides a robust solution to this problem and is effective in extending the applicability range of the turbidity methodology, as well as increasing measurement accuracy. This paper will provide a more comprehensive discussion of the physical insight for using the T-matrix method in turbidity measurement and associated technical details. In particular, the importance of ensuring the correct value for the refractive indices for colloidal particles and the surrounding medium used in the calculation is addressed, because the indices generally vary with the wavelength of the incident light. The comparison of calculated results with experiments shows that the T-matrix method can correctly calculate optical factors even for large particles, whereas other existing theories cannot. In addition, the data of the optical factor calculated by the T-matrix method for a range of particle radii and incident light wavelengths are listed.
\end{abstract}

(c) 2006 Elsevier Inc. All rights reserved.

Keywords: Coagulation; Turbidity measurement; Absolute coagulation rate constant; Optical factor; T-matrix method

\section{Introduction}

In turbidity measurement, the rate of turbidity change due to the aggregation of particles is measured, and the absolute aggregation rate is related to the rate of turbidity change through the so-called optical factor, which has to be calculated by means of light scattering theory. The relationship of the absolute aggregation rate constant with the measured turbidity changing rate can be described as follows [1],

$k_{\text {aggr }}=\frac{\left(1 / \tau_{0}\right)(\mathrm{d} \tau / \mathrm{d} t)_{0}}{\left[C_{2} / 2 C_{1}-1\right] N_{1}}$,

where $k_{\text {aggr }}$ is the absolute aggregation rate constant, $\tau_{0}$ is the turbidity at the beginning of aggregation, $(\mathrm{d} \tau / \mathrm{d} t)_{0}$ is the turbidity change rate at the beginning of aggregation, and $N_{1}$ is the number concentration of particles in the monodisperse suspensions. The dimensionless parameter $F=\left[\left(C_{2} / 2 C_{1}\right)-1\right]$ is

\footnotetext{
* Corresponding author.

E-mail address: sunzw@imech.ac.cn (Z.Sun).
}

referred to as the optical factor, where $C_{2}$ and $C_{1}$ are the extinction cross sections of two aggregated particles and one single particle, respectively.

Because the optical factor $F$ cannot be measured experimentally, a major problem in turbidity measurement is how to calculate $C_{1}$ and $C_{2}$ theoretically. Mie theory and RayleighGans-Debye (RGD) theory have been employed to calculate the optical factor. In a previous paper [2], we have shown that existing theories are not applicable to large particles and only the T-matrix method is capable of extending the applicability range of the turbidity methodology and increasing measurement accuracy. Although an outline of using the T-matrix to evaluate the extinction cross section was described in Ref. [2], the application of the T-matrix method to optical factor calculation is not straightforward for practical implementation. We found that how to evaluate the relative refractive index of particle and surrounding medium, which varies with the incident wavelength, is a crucial element in corrected calculation of the optical factor, but this part was not discussed, in order not to hinder the previous paper from its major theme. 
The main focus of this paper is on offering more details on using the T-matrix to calculate the optical factor. We give special attention to the importance of ensuring the correct value of the refractive indices for the liquid phase and colloidal particle used in the calculation of optical factors, because the indices generally vary with the incident wavelength. The optical factors calculated by the T-matrix method are compared with other existing theories and experiments. The factors causing uncertainty in determination of the turbidity measurement for the absolute coagulation rate constant are estimated, and advice on how to improve the accuracy of the measurement is presented. Finally, to make it possible for interested readers to easily test the T-matrix method in turbidity measurement, we list data of optical factors calculated by the T-matrix method for a range of particle radii and incident light wavelengths.

\section{Theory}

\subsection{Calculation of optical factors by Mie theory and RGD theory}

The Mie theory is valid for calculating the cross sections for spheres of any size [3,4]. Therefore it can be used to evaluate $C_{1}$ accurately. The Mie theory, however, cannot evaluate $C_{2}$ correctly because it is associated with the extinction cross section (or scattering cross section for a nonabsorbing particle) of a doublet composed of two spheres. One way to overcome this difficulty is to adopt the coalescing assumption for a doublet, in which the aggregated doublet is considered to coalesce into a spherical particle with the same volume as the two separated particles. However, the coalescence does not actually take place, so the error associated with this coalescence assumption is unavoidable in the calculation of the optical factor. We will refer to this approximation method as Mie (coalescence) below.

Another approximation for dealing with the scattering problems of particles is the Rayleigh-Gans-Debye (RGD) theory. In this theory, the whole volume of the scattering object is subdivided into many volume elements, and each element represents a Rayleigh scatterer. As it is developed from Rayleigh theory, RGD theory is only valid for small particles. Besides single spherical particles, the RGD theory is also applicable to real doublets. Therefore, RGD theory can be used to evaluate the optical factors of small particles. We will refer to this method as RGD (real).

The RGD method can also be used to calculate the optical factor with the coalescence assumption. We will call this method RGD (coalescence). Using the results of Mie (coalescence) and RGD (coalescence), Lichtenbelt et al. [1] have tried to improve the calculation of the optical factor by correcting the RGD (real) results. The corrected optical factor is calculated using the formula

$\frac{C_{2}}{2 C_{1}}-1=\frac{C_{2}^{\prime}(\mathrm{Mie}) C_{2}(\mathrm{RGD})}{2 C_{1}(\mathrm{Mie}) C_{2}^{\prime}(\mathrm{RGD})}-1$,

where $C_{2}^{\prime}$ (Mie), $C_{2}$ (RGD), and $C_{2}^{\prime}$ (RGD) are calculated from Mie (coalescence), RGD (real), and RGD (coalescence), re- spectively. $C_{1}$ (Mie) is calculated from Mie theory. We will refer to this method as RGD (corrected).

It can be seen that all the methods mentioned above either cannot treat large particles correctly or have to resort to the coalescing assumption in calculating the optical factors. Thus all these existing theories may give incorrect optical factors for large particles.

\subsection{T-matrix method}

The superiority of the T-matrix method [5-9] over other approaches discussed above is that it can accurately solve more complex scattering problems for irregular particles, including aggregates composed of spherical particles [9-13]. There is no size limitation (unlike RGD theory, which is limited to small particles) or shape restriction (unlike Mie theory, which is limited to spherical particles) on particles as a prerequisite to this theory. Therefore, the T-matrix method provides powerful tools for accurate calculation of the optical factors without limitations on particle size.

In the T-matrix method, both incident and scattered electric fields are expanded in a series of vector spherical wave functions as follows [9]

$$
\begin{aligned}
& \mathbf{E}^{\mathrm{inc}}(\mathbf{r})=\sum_{n=1}^{\infty} \sum_{m=-n}^{n}\left[a_{m n} \operatorname{Rg} \mathbf{M}_{m n}(k \mathbf{r})+b_{m n} \operatorname{Rg} \mathbf{N}_{m n}(k \mathbf{r})\right], \\
& \mathbf{E}^{\mathrm{sca}}(\mathbf{r})=\sum_{n=1}^{\infty} \sum_{m=-n}^{n}\left[p_{m n} \mathbf{M}_{m n}(k \mathbf{r})+q_{m n} \mathbf{N}_{m n}(k \mathbf{r})\right],
\end{aligned}
$$

where $k=2 \pi / \lambda$, and $\lambda$ is the wavelength. The harmonics $\operatorname{Rg} \mathbf{M}_{m n}$ and $\operatorname{Rg} \mathbf{N}_{m n}$ have a Bessel-function radial dependence and are regular (finite) at the origin, whereas the functions $\mathbf{M}_{m n}$ and $\mathbf{N}_{m n}$ have a Hankel-function radial dependence and vanish at infinity. Due to the linearity of Maxwell's equations, the scattered field coefficients $\mathbf{p}=\left[p_{m n}, q_{m n}\right]$ are related to the incident field coefficients $\mathbf{a}=\left[a_{m n}, b_{m n}\right]$ by means of the so-called transition matrix (or T-matrix):

$$
\begin{aligned}
& p_{m n}=\sum_{n^{\prime}=1}^{\infty} \sum_{m^{\prime}=-n^{\prime}}^{n^{\prime}}\left[T_{m n m^{\prime} n^{\prime}}^{11} a_{m^{\prime} n^{\prime}}+T_{m n m^{\prime} n^{\prime}}^{12} b_{m^{\prime} n^{\prime}}\right], \\
& q_{m n}=\sum_{n^{\prime}=1}^{\infty} \sum_{m^{\prime}=-n^{\prime}}^{n^{\prime}}\left[T_{m n m^{\prime} n^{\prime}}^{21} a_{m^{\prime} n^{\prime}}+T_{m n m^{\prime} n^{\prime}}^{22} b_{m^{\prime} n^{\prime}}\right] .
\end{aligned}
$$

In compact matrix notation, Eqs. (5) and (6) can be rewritten as

$\left[\begin{array}{l}\mathbf{p} \\ \mathbf{q}\end{array}\right]=\mathbf{T}\left[\begin{array}{l}\mathbf{a} \\ \mathbf{b}\end{array}\right]=\left[\begin{array}{ll}\mathbf{T}^{11} & \mathbf{T}^{12} \\ \mathbf{T}^{21} & \mathbf{T}^{22}\end{array}\right]\left[\begin{array}{l}\mathbf{a} \\ \mathbf{b}\end{array}\right]$,

which means that the column vector of the expansion coefficients of the scattered field is obtained by multiplying the transition matrix $(\mathbf{T})$ and the column vector of the expansion coefficients of the incident field.

For a spherical particle, the T-matrix is diagonal, and its elements are simply the $a_{n}$ and $b_{n}$ coefficients from Mie scattering [4]. Thus the T-matrix method converges with Mie theory for a single spherical particle. However, the complex T-matrix 
method can be used to calculate the light-scattering properties of nonspherical and composite scatterers, particularly bispheres. Therefore, it is capable of calculating the scattering cross section of doublet $C_{2}$ with no additional assumptions, such as those involved in conventional Mie theory and RGD theory.

Consider now the computation of the T-matrix for a cluster consisting of $N$ spheres. The total scattered electric field can be written as the sum of the fields scattered by all spheres:

$\mathbf{E}^{\mathrm{sca}}(\mathbf{r})=\sum_{j=1}^{N} \mathbf{E}_{j}^{\mathrm{sca}}(\mathbf{r})$.

Because of the electromagnetic interactions between the components, the total electric field exciting each particle can be written as the sum of the external incident field $\mathbf{E}_{0}^{\text {inc }}(\mathbf{r})$ and the partial fields scattered by all other particles:

$\mathbf{E}_{j}^{\mathrm{inc}}(\mathbf{r})=\mathbf{E}_{0}^{\mathrm{inc}}(\mathbf{r})+\sum_{\substack{l=1 \\ l \neq j}}^{N} \mathbf{E}_{l}^{\mathrm{sca}}(\mathbf{r}), \quad j=1,2, \ldots, N$.

To make use of the information contained in the $j$ th component of the T-matrix, the fields incident on and scattered by this component can be expanded in vector spherical wave functions centered at the origin of the component's local coordinate system,

$$
\begin{aligned}
\mathbf{E}_{j}^{\mathrm{inc}}(\mathbf{r})= & \sum_{n=1}^{\infty} \sum_{m=-n}^{n}\left[a_{m n}^{j} \operatorname{Rg} \mathbf{M}_{m n}\left(k \mathbf{r}_{j}\right)+b_{m n}^{j} \operatorname{Rg} \mathbf{N}_{m n}\left(k \mathbf{r}_{j}\right)\right] \\
= & \sum_{n=1}^{\infty} \sum_{m=-n}^{n}\left[\left(a_{m n}^{j 0}+\sum_{l \neq j} a_{m n}^{j l}\right) \operatorname{Rg} \mathbf{M}_{m n}\left(k \mathbf{r}_{j}\right)\right. \\
& \left.+\left(b_{m n}^{j 0}+\sum_{l \neq j} b_{m n}^{j l}\right) \operatorname{Rg}_{m n}\left(k \mathbf{r}_{j}\right)\right], \\
\mathbf{E}_{j}^{\mathrm{sca}}(\mathbf{r})= & \sum_{n=1}^{\infty} \sum_{m=-n}^{n}\left[p_{m n}^{j} \mathbf{M}_{m n}\left(k \mathbf{r}_{j}\right)+q_{m n}^{j} \mathbf{N}_{m n}\left(k \mathbf{r}_{j}\right)\right], \\
j=1, & 2, \ldots, N,
\end{aligned}
$$

where the index $j$ numbers the spheres and the vector $\mathbf{r}_{j}$ originates at the center of the $j$ th sphere. The coefficients $a_{m n}^{j 0}$ and $b_{m n}^{j 0}$ describe the external incident field, and the expansion coefficients $a_{m n}^{j l}$ and $b_{m n}^{j l}$ describe the contribution of the $l$ th component to the field illuminating the $j$ th component.

Similarly to Eq. (7), the expansion coefficients of Eqs. (10) and (11) are related via the $j$ th component of the T-matrix $\mathbf{T}^{j}$ :

$$
\left[\begin{array}{l}
\mathbf{p}^{j} \\
\mathbf{q}^{j}
\end{array}\right]=\mathbf{T}^{j}\left(\left[\begin{array}{l}
\mathbf{a}^{j 0} \\
\mathbf{b}^{j 0}
\end{array}\right]+\sum_{l \neq j}\left[\begin{array}{l}
\mathbf{a}^{j l} \\
\mathbf{b}^{j l}
\end{array}\right]\right) .
$$

As shown in Ref. [9], the vector spherical wave functions appearing here can be expanded in regular vector spherical wave functions centered at the origin of the $j$ th reference frame,

$$
\begin{aligned}
\mathbf{M}_{\mu \nu}\left(k \mathbf{r}_{l}\right)= & \sum_{n=1}^{\infty} \sum_{m=-n}^{n}\left[A_{m n \mu \nu}\left(k \mathbf{r}_{l j}\right) \operatorname{Rg} \mathbf{M}_{m n}\left(k \mathbf{r}_{j}\right)\right. \\
& \left.+B_{m n \mu \nu}\left(k \mathbf{r}_{l j}\right) \operatorname{Rg} \mathbf{N}_{m n}\left(k \mathbf{r}_{j}\right)\right]
\end{aligned}
$$

$$
\begin{aligned}
\mathbf{N}_{\mu \nu}\left(k \mathbf{r}_{l}\right)= & \sum_{n=1}^{\infty} \sum_{m=-n}^{n}\left[B_{m n \mu \nu}\left(k \mathbf{r}_{l j}\right) \operatorname{Rg} \mathbf{M}_{m n}\left(k \mathbf{r}_{j}\right)\right. \\
& \left.+A_{m n \mu v}\left(k \mathbf{r}_{l j}\right) \operatorname{Rg} \mathbf{N}_{m n}\left(k \mathbf{r}_{j}\right)\right],
\end{aligned}
$$

where the vector $\mathbf{r}_{l j}=\mathbf{r}_{l}-\mathbf{r}_{j}$. The explicit expressions for the translation coefficients $A_{\text {mnuv }}\left(k \mathbf{r}_{l j}\right)$ and $B_{\text {mnuv }}\left(k \mathbf{r}_{l j}\right)$ can be found in Ref. [9]. Thus we can write Eq. (12) as

$$
\begin{aligned}
{\left[\begin{array}{l}
\mathbf{p}^{j} \\
\mathbf{q}^{j}
\end{array}\right] } & =\mathbf{T}^{j}\left(\left[\begin{array}{l}
\mathbf{a}^{j 0} \\
\mathbf{b}^{j 0}
\end{array}\right]+\sum_{l \neq j}\left[\begin{array}{ll}
\mathbf{A}\left(k \mathbf{r}_{l j}\right) & \mathbf{B}\left(k \mathbf{r}_{l j}\right) \\
\mathbf{B}\left(k \mathbf{r}_{l j}\right) & \mathbf{A}\left(k \mathbf{r}_{l j}\right)
\end{array}\right]\left[\begin{array}{l}
\mathbf{p}^{l} \\
\mathbf{q}^{l}
\end{array}\right]\right) \\
& =\mathbf{T}^{j}\left(\left[\begin{array}{l}
\mathbf{a}^{j 0} \\
\mathbf{b}^{j 0}
\end{array}\right]+\sum_{l \neq j} \mathbf{A}^{j l}\left[\begin{array}{l}
\mathbf{p}^{l} \\
\mathbf{q}^{l}
\end{array}\right]\right),
\end{aligned}
$$

where $\mathbf{T}^{j}$ represents the T-matrix for the particle $j$, when isolated. Inversion of Eq. (15) gives sphere-centered transition matrices that transform the expansion coefficients of the incident field into expansion coefficients of the individual scattered fields $[9,12]$,

$\left[\begin{array}{l}\mathbf{p}^{j} \\ \mathbf{q}^{j}\end{array}\right]=\sum_{l=1}^{N} \mathbf{T}^{j l}\left[\begin{array}{l}\mathbf{a}^{l 0} \\ \mathbf{b}^{l 0}\end{array}\right], \quad j=1,2, \ldots, N$,

where the matrix $\mathbf{T}^{j l}$ transforms the coefficients of the incident field expansion centered at the $l$ th origin into expansion coefficients of the partial field scattered by the $j$ th component at the $j$ th origin.

The expansion coefficients for the incident field at the origin of sphere $i,\left(\mathbf{a}^{i 0}, \mathbf{b}^{i 0}\right)$, can be obtained by a translation of the incident field coefficients expanded about the cluster origin, $(\mathbf{a}, \mathbf{b})$, to origin $i$ through $[9,12]$

$\left[\begin{array}{l}\mathbf{a}^{i 0} \\ \mathbf{b}^{i 0}\end{array}\right]=\mathbf{B}^{i}\left[\begin{array}{l}\mathbf{a} \\ \mathbf{b}\end{array}\right]$,

where the $\mathbf{B}$ matrices are similar to the $\mathbf{A}$ matrices of Eq. (15).

In a similar fashion, the combined scattered field from all the spheres can be expressed as a single expansion written about the cluster origin by a translation of the fields from the $j$ th spheres origin to the cluster origin. The expansion coefficients representing the total scattered field are given by Refs. [9,12]:

$\left[\begin{array}{l}\mathbf{p} \\ \mathbf{q}\end{array}\right]=\sum_{j} \mathbf{B}^{j}\left[\begin{array}{l}\mathbf{p}^{j} \\ \mathbf{q}^{j}\end{array}\right]$.

When Eqs. (16)-(18) are combined, the scattered field expansions from the individual spheres will be transformed into a single expansion based on a single origin of the particle system. The incident and scattered coefficients for the system will be derived as $[9,10,13]$

$$
\begin{aligned}
{\left[\begin{array}{l}
\mathbf{p} \\
\mathbf{q}
\end{array}\right] } & =\sum_{j} \mathbf{B}^{j}\left[\begin{array}{l}
\mathbf{p}^{j} \\
\mathbf{q}^{j}
\end{array}\right]=\sum_{j, i} \mathbf{B}^{j} \mathbf{T}^{j i}\left[\begin{array}{l}
\mathbf{a}^{i 0} \\
\mathbf{b}^{i 0}
\end{array}\right]=\sum_{j, i} \mathbf{B}^{j} \mathbf{T}^{j i} \mathbf{B}^{i}\left[\begin{array}{l}
\mathbf{a} \\
\mathbf{b}
\end{array}\right] \\
& =\mathbf{T}\left[\begin{array}{l}
\mathbf{a} \\
\mathbf{b}
\end{array}\right]=\left[\begin{array}{ll}
\mathbf{T}^{\mathbf{1 1}} & \mathbf{T}^{\mathbf{1 2}} \\
\mathbf{T}^{\mathbf{1 1}} & \mathbf{T}^{\mathbf{2 2}}
\end{array}\right]\left[\begin{array}{l}
\mathbf{a} \\
\mathbf{b}
\end{array}\right] .
\end{aligned}
$$

The matrix $\mathbf{T}$ defined in Eq. (19) is the T-matrix that we seek and can be used directly in computing the orientationally averaged scattering matrix for the aggregated cluster. As in our 
paper, what we need is to calculate the scattering cross section of two aggregated particles $C_{2}$, in this case $N=2$. The explicit expressions and calculations for the T-matrix can be found in Ref. [9].

After getting the T-matrix of two aggregated particles, we can obtain the extinction cross section by the formula given in Ref. [9]:

$\left\langle C_{\text {ext }}\right\rangle=-\frac{2 \pi}{k^{2}} \operatorname{Re} \sum_{n=1}^{\infty} \sum_{m=-n}^{n}\left[T_{m n m n}^{11}+T_{m n m n}^{22}\right]$.

By this means, the extinction cross section of two aggregated particles $C_{2}$ can be calculated by the T-matrix method, without any further assumptions as required by Mie theory or RGD theory. The extinction cross section of a single particle $C_{1}$ can be calculated by Mie theory or by the above-mentioned T-matrix method. And then the optical factors can be determined from the results of $C_{2}$ and $C_{1}$.

\section{Wavelength correction of the refractive index used in the T-matrix method}

Although the deduction of the T-matrix for the extinction cross section of two aggregated particles may seem complicated, only two parameters are required for the calculation of the elements of the T-matrix (for a single particle or the aggregates of two particles), namely, the size parameter $x=2 \pi a / \lambda_{1}$ and the relative refractive index $m=n_{2} / n_{1}$, where $a$ is the radius of a single particle, $\lambda_{1}$ is the wavelength in the medium, and $n_{2}$ and $n_{1}$ are the refractive indices of the particle and the surrounding medium, respectively. Both $x$ and $m$ are also required for the calculation of optical factors by RGD theory and Mie theory.

It is elementary knowledge that the refractive index changes with the wavelength of the incident light. However, for polystyrene particles dispersed in water, as used in this study, fixed values of $n_{1}=1.33$ and $n_{2}=1.60$ are usually adopted in the determination of cross sections and optical factors $[1,13]$, because the difference of refractive indices for different wavelengths is not significant. In this case, $m$ is considered to be a constant and then the size parameter $x$ becomes the only variable for the optical factor. Lichtenbelt et al. [1] published their data of optical factors calculated by Mie theory and RGD theory, with only one variable $x$. In their results by Mie (coalescence) and RGD (corrected), $m$ is taken to be a constant 1.20 in value, while for RGD (coalescence) and RGD (corrected), $m$ is no longer a parameter in the expression because of the cancellation for $m$ contained in $C_{1}$ and $C_{2}$ in Eq. (1). Therefore RGD (both coalescence and corrected) does not depend on $m$; the size parameter $x$ as the only variable should be sufficient in the calculation of the optical factor.

As has been shown $[1,2,4]$, when the size parameter $x$ is small, RGD theory provides a reasonable approximation. Since other theories (including the T-matrix method) can yield optical factors that are basically equivalent to those from $m$-independent RGD theory, other theories with a constant $m$ or taking $x$ to be the only parameter should not introduce considerable error into the calculation of the optical factor as long as $x$ is small. In this case, the correction to $m$ has little influence on the calculation of the optical factor. However, our experiments show that using only one variable, $x$, to characterize the optical factor is insufficient, at least for the case with a large size parameter.

Equation (1) actually suggests an experimental approach to testing whether a theory is effective for delivering a good optical factor. From Eq. (1) we can see that

$$
\left(1 / \tau_{0}\right)(\mathrm{d} \tau / \mathrm{d} t)_{0} / N_{1}=\left[C_{2} / 2 C_{1}-1\right] k_{\mathrm{aggr}} .
$$

Using $R$ to denote the experimentally measured quantity $\left(1 / \tau_{0}\right)(\mathrm{d} \tau / \mathrm{d} t)_{0} / N_{1}$, we will have $R=F k_{\text {aggr. }}$. As $k_{\text {aggr }}$ is a constant for a given colloid system, the $F$ must be proportional to $R$. Fig. 1 shows the experimentally measured $R$ with the size parameter $x$ for particles of $a=250$ and $500 \mathrm{~nm}$, respectively. The experiments were performed with a UV-vis dual-beam spectrophotometer (Purkinje TU-1901, Beijing), as described in Ref. [2]. This plot shows that the $R \sim x$ curve for particles with $a=250 \mathrm{~nm}$ has very different behavior from that for particles with $a=500 \mathrm{~nm}$, implying that $R$ depends not only on the size parameter $x$ but also on the particle size itself. Therefore we conclude that using a single variable $x$ in a theoretical formulation is not sufficient to determine the optical factor correctly.

Actually, to keep $x$ unchanged, the wavelength has to be altered as different particle sizes are used. If the correction of refractive indices with $\lambda$ is considered, the calculated optical factors may be different for different particle sizes even with a fixed $x$, as in the results of Fig. 1. Therefore, correction of refractive indices is essential in determining the optical factor.

The dependence of the refractive indices of water and polystyrene on wavelength has been studied [14-19]. The refractive index of water is a function of the wavelength $\lambda$, salinity $S$, and temperature $T$ [14-16]. The empirical formulas of the refractive index of water in relation to wavelength given by the references [14-16] are different, but the results are quite similar. In this study, we will use the formulas given by

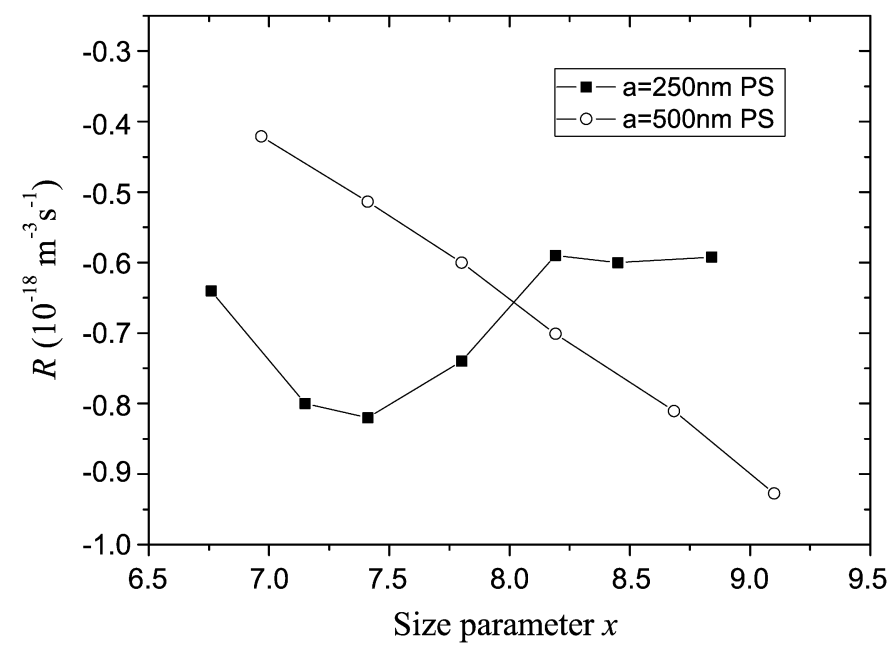

Fig. 1. Plot of $R=\left(1 / \tau_{0}\right)(\mathrm{d} \tau / \mathrm{d} t)_{0} / N_{1}$ changes with $x$ for different-sized particles $(a=250$ and $500 \mathrm{~nm})$. 


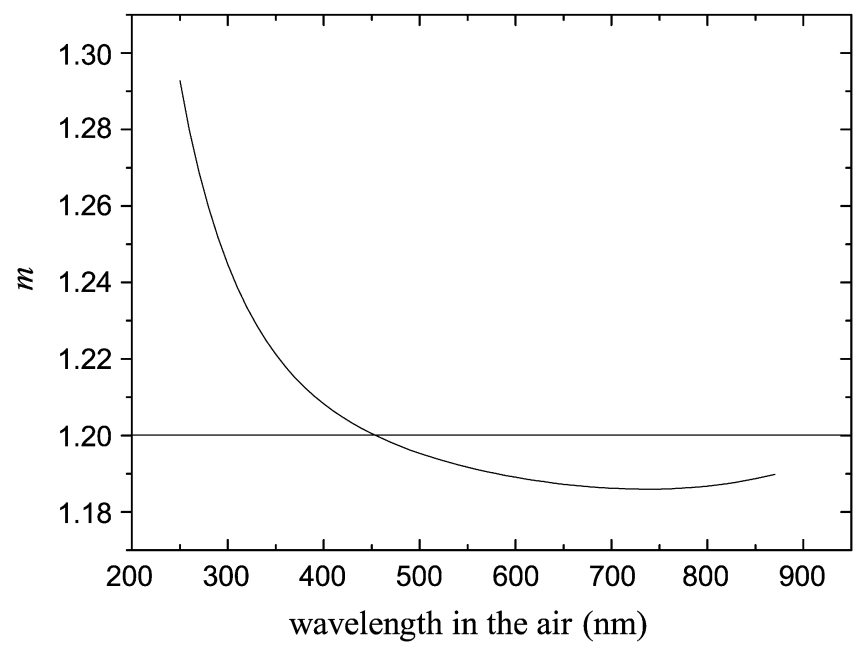

Fig. 2. $m$ corrected using Eqs. (21) and (22) with $T=25^{\circ} \mathrm{C}$ and $S=28$ for different wavelengths available from spectrophotometer.

Ref. [16],

$$
\begin{aligned}
n_{\text {water }}(\lambda)= & 1.447824+3.0110 \times 10^{-4} S-1.8029 \times 10^{-5} T \\
& -1.6916 \times 10^{-6} T^{2}-4.89040 \times 10^{-1} \lambda \\
& +7.28364 \times 10^{-1} \lambda^{2}-3.83745 \times 10^{-1} \lambda^{3} \\
& -S\left(7.9362 \times 10^{-7} T-8.0597 \times 10^{-9} T^{2}\right. \\
& +4.249 \times 10^{-4} \lambda-5.847 \times 10^{-4} \lambda^{2} \\
& \left.+2.812 \times 10^{-4} \lambda^{3}\right),
\end{aligned}
$$

where $S$ is the salinity in $\%, T$ is the temperature in degrees Celsius, and $\lambda$ is the wavelength in micrometers.

References [17-19] present an empirical formula for the polystyrene refractive index. However, we have noticed that the results of Ref. [17] are slightly different from the data of Refs. $[18,19]$. In this paper, we use the data given by Ref. [19], and fit the data to the Cauchy dispersion relation as

$n_{\mathrm{ps}}(\lambda)=1.56362+0.00884 / \lambda^{2}+0.000255627 / \lambda^{4}$,

where the unit for wavelength is micrometers.

By using Eqs. (21) and (22), we can obtain the corrected values for the refractive indices of water and polystyrene for different wavelengths to get the parameters $x$ and $m$ required in the calculation of optical factors. The plot in Fig. 2 is the wavelength-dependence curve of the corrected relative refractive index $m$ using Eqs. (21) and (22), where $T=25^{\circ} \mathrm{C}$ and $S=28$. Since the wavelength of the incident light mentioned here is supposed to be measured in air but $\lambda_{1}$ is considered to be the wavelength in water, the size parameter $x=2 \pi a / \lambda_{1}$ is also related to the refractive index of water. Fig. 3 shows the size parameter $x$ of a particle with radius $500 \mathrm{~nm}$ for different wavelengths calculated by using the corrected and constant refractive index of water. The difference between the corrected $m$ and constant $m=1.20$ is small ( $<8 \%$ ) for the wavelength available from a UV-vis spectrophotometer, and the differences in the size parameter $x$ are even smaller.

However, the differences in optical factors $F$ calculated by the T-matrix method with corrected refractive indices and constant indices are very different, as shown in Fig. 4 for particles

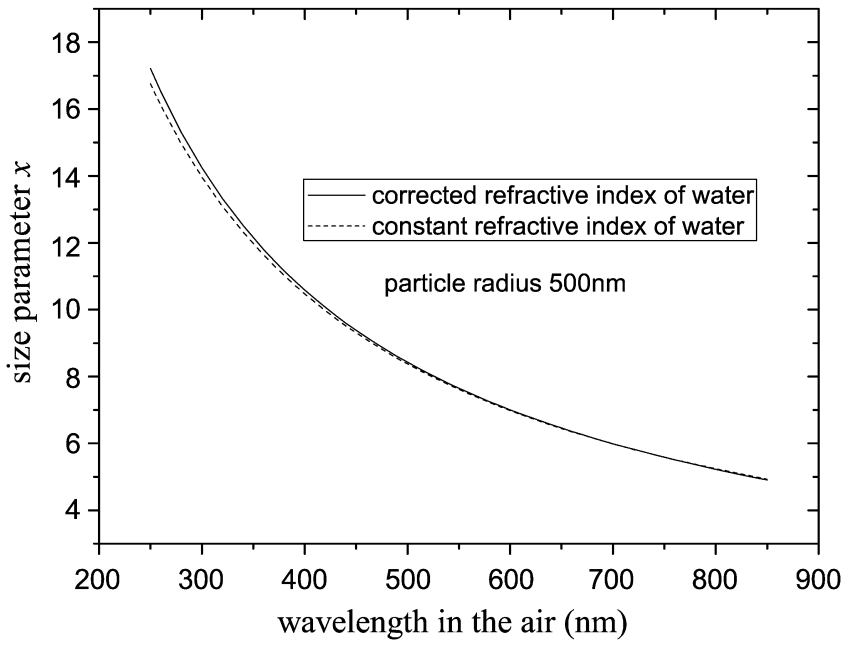

Fig. 3. The size parameter $x$ calculated from constant refractive index and corrected refractive index of water using Eq. (21) with $T=25^{\circ} \mathrm{C}$ and $S=28$ for different wavelengths available from spectrophotometer. The particle radius is $500 \mathrm{~nm}$.

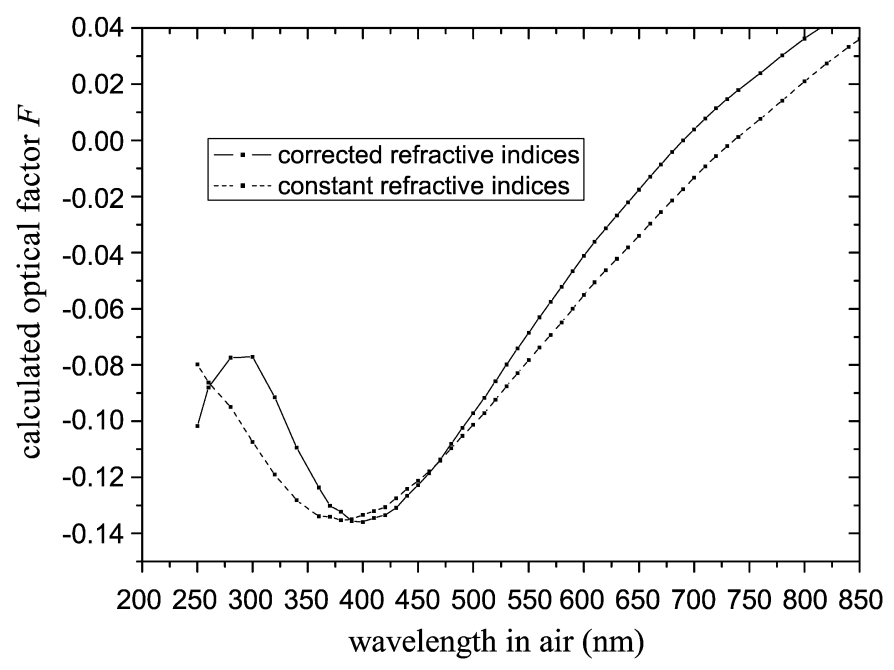

Fig. 4. The optical factors $F$ calculated by the T-matrix method with constant refractive indices and corrected refractive indices using Eqs. (21) and (22) with $T=25^{\circ} \mathrm{C}$ and $S=28$. The particle radius is $500 \mathrm{~nm}$.

with radius $500 \mathrm{~nm}$. It can be seen that even the shapes and the changing tendencies of both curves are quite different. Therefore, small corrections to $x$ and $m$ may result in significant differences in the calculated optical factor $F$. To more clearly show the importance of ensuring the corrected refractive indices used in the calculation of optical factors, Table 1 lists the percentage differences in $m, x$, and calculated $F$, respectively, corresponding to the corrected and the constant indices. Again these data provide direct evidence that if the relevant corrections to refractive indices are not made, large errors may appear, even though there is nothing wrong with the T-matrix method itself.

\section{Results and discussion}

We use the fact that the optical factor $F$ is proportional to $R$ at different wavelengths to examine the performance of a 
Table 1

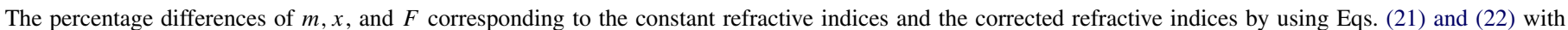
$T=25^{\circ} \mathrm{C}$ and $S=28^{\mathrm{a}}$

\begin{tabular}{|c|c|c|c|c|c|c|c|c|c|c|c|}
\hline$\lambda$ in the air (nm) & 300 & 340 & 380 & 420 & 500 & 550 & 600 & 650 & 740 & 800 & 820 \\
\hline$\Delta m / m(\%)$ & 3.59 & 2.02 & 1.04 & 0.39 & 0.39 & 0.70 & 0.92 & 1.07 & 1.18 & 1.11 & 1.06 \\
\hline$\Delta x / x(\%)$ & 2.00 & 1.58 & 1.24 & 0.96 & 0.58 & 0.42 & 0.30 & 0.19 & 0.04 & 0.27 & 0.37 \\
\hline$\Delta F / F(\%)$ & 39.35 & 17.11 & 2.23 & 2.13 & 4.30 & 14.28 & 33.93 & 93.93 & 92.82 & 42.42 & 34.14 \\
\hline
\end{tabular}

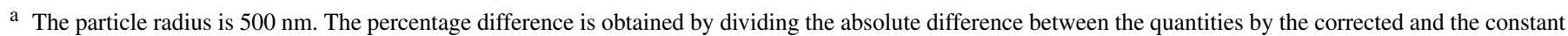
relative refractive indices by the former.

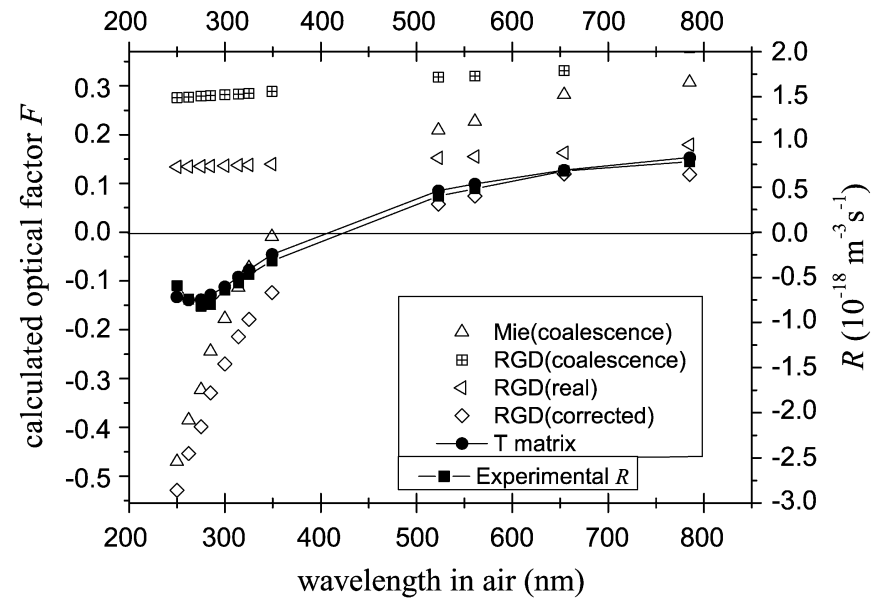

(a)

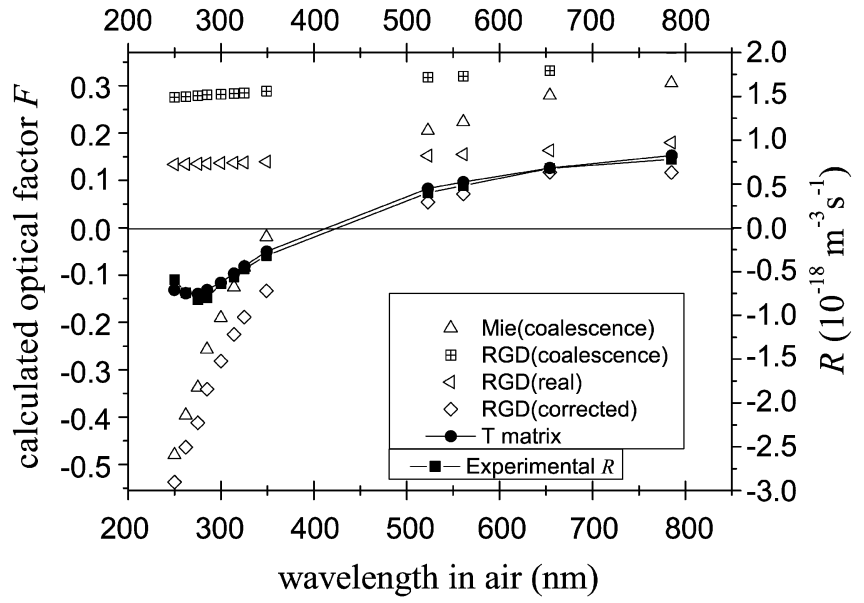

(b)

Fig. 5. The optical factors calculated for different size parameters using the T-matrix method and the Mie (coalescence), RGD (coalescence), RGD (real), and RGD (corrected) methods. The experimental results of $R=\left(1 / \tau_{0}\right)(\mathrm{d} \tau / \mathrm{d} t)_{0} / N_{1}$ are also compared in the figure. The radius of dispersed particles is $250 \mathrm{~nm}$. (a) The refractive indices of water and polystyrene are calculated from Eqs. (21) and (22) with $S=28$ and $T=25^{\circ} \mathrm{C}$. (b) The refractive indices of water and polystyrene are calculated from Eqs. (21) and (22) with $S=0$ and $T=25^{\circ} \mathrm{C}$.

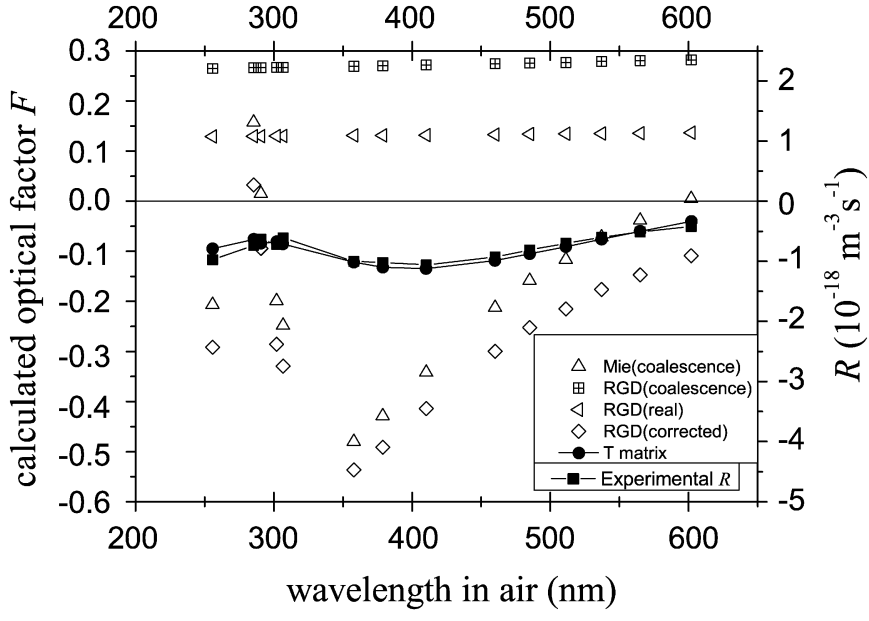

(a)

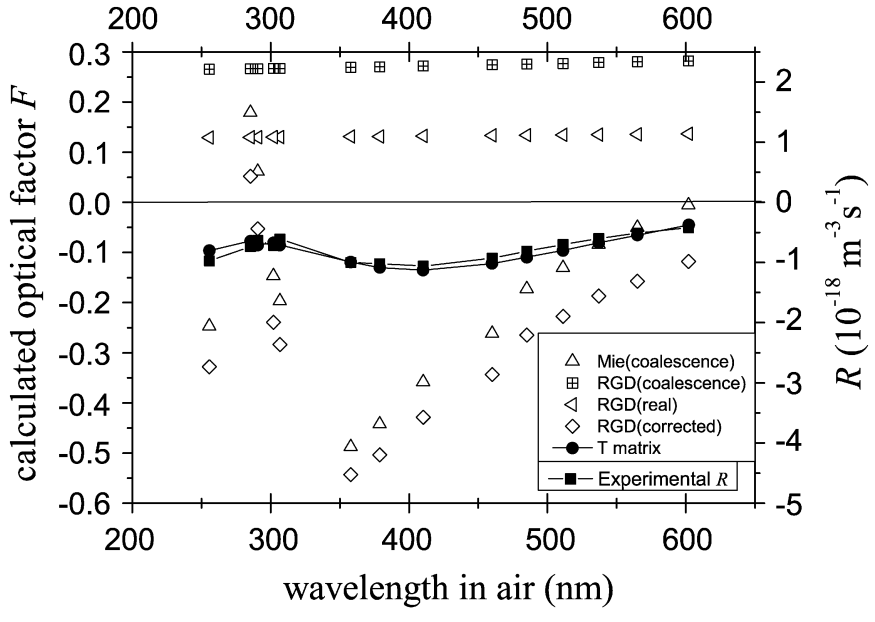

(b)

Fig. 6. The optical factors for different size parameters calculated using the T-matrix method and the Mie (coalescence), RGD (coalescence), RGD (real), and RGD (corrected) methods. The experimental results of $R=\left(1 / \tau_{0}\right)(\mathrm{d} \tau / \mathrm{d} t)_{0} / N_{1}$ are also compared in the figure. The radius of dispersed particles is $500 \mathrm{~nm}$. (a) The refractive indices of water and polystyrene are calculated from Eqs. (21) and (22) with $S=28$ and $T=25^{\circ} \mathrm{C}$. (b) The refractive indices of water and polystyrene are calculated from Eqs. (21) and (22) with $S=0$ and $T=25^{\circ} \mathrm{C}$.

theory in the calculation of the optical factor, by comparing the results of $R$ at different wavelengths with the optical factor calculated by the theory. For a good theory, the calculated optical factors should basically coincide with the measured $R$ for different wavelengths, after an appropriate scaling factor is multiplied by the vertical coordinate axis.
The results of the optical factors from different theories and the experimental results of $R$ versus incident wavelength in the air are compared in Figs. 5 and 6.

Both Figs. 5a and 5b show optical factors calculated by different theories compared with the experimental results of $R$ for $a=250$-nm PS particles at $T=25^{\circ} \mathrm{C}$. But the data in Fig. 5a 
Table 2

Optical factors for some typical wavelengths (in air) and particle sizes calculated by the T-matrix method ${ }^{\mathrm{a}}$

\begin{tabular}{|c|c|c|c|c|c|c|c|c|}
\hline \multirow{2}{*}{$\begin{array}{l}\lambda \\
(\mathrm{nm})\end{array}$} & \multicolumn{8}{|c|}{ Diameter of particle $(\mathrm{nm})$} \\
\hline & 100 & 200 & 300 & 400 & 500 & 600 & 800 & 1000 \\
\hline 850 & 0.760141 & 0.351878 & 0.251218 & 0.212014 & 0.165216 & 0.140811 & 0.09319 & 0.048585 \\
\hline 780 & 0.718692 & 0.307114 & 0.245647 & 0.195708 & 0.152869 & 0.127339 & 0.077675 & 0.030255 \\
\hline 700 & 0.660885 & 0.270121 & 0.228921 & 0.170864 & 0.13887 & 0.109268 & 0.055966 & 0.00395 \\
\hline 633 & 0.601367 & 0.255205 & 0.209394 & 0.15355 & 0.122053 & 0.091348 & 0.031181 & -0.02537 \\
\hline 600 & 0.567621 & 0.252705 & 0.199244 & 0.147412 & 0.112335 & 0.079757 & 0.016531 & -0.04116 \\
\hline 532 & 0.488165 & 0.247933 & 0.170957 & 0.126896 & 0.088889 & 0.05159 & -0.01956 & -0.07869 \\
\hline 500 & 0.446574 & 0.240342 & 0.157603 & 0.115612 & 0.07411 & 0.034873 & -0.03959 & -0.09713 \\
\hline 460 & 0.392351 & 0.224137 & 0.146353 & 0.097269 & 0.053074 & 0.008718 & -0.06809 & -0.11859 \\
\hline 441 & 0.36665 & 0.215148 & 0.139692 & 0.088317 & 0.040972 & -0.00442 & -0.08194 & -0.12619 \\
\hline 400 & 0.315189 & 0.194204 & 0.118938 & 0.062058 & 0.008464 & -0.04076 & -0.11191 & -0.13587 \\
\hline 350 & 0.27207 & 0.156328 & 0.084023 & 0.017581 & -0.04415 & -0.09302 & -0.13717 & -0.11684 \\
\hline 337 & 0.266588 & 0.148101 & 0.073394 & 0.00206 & -0.06117 & -0.10652 & -0.13544 & -0.10527 \\
\hline 325 & 0.263712 & 0.14196 & 0.06153 & -0.01511 & -0.07659 & -0.11925 & -0.1337 & -0.09758 \\
\hline 300 & 0.262268 & 0.12331 & 0.027225 & -0.05248 & -0.11185 & -0.13738 & -0.114 & -0.07711 \\
\hline 275 & 0.256605 & 0.091826 & -0.01616 & -0.09916 & -0.13841 & -0.13437 & -0.08009 & -0.08411 \\
\hline 250 & 0.231062 & 0.045079 & -0.07658 & -0.13857 & -0.13273 & -0.09715 & -0.07984 & -0.10171 \\
\hline
\end{tabular}

a The refractive indices of water and polystyrene are calculated using Eqs. (21) and (22), where $S=28$ and $T=25^{\circ} \mathrm{C}$ are used in the calculation.

corresponding to salinity $S=28$ (the case in our experiment), while those in Fig. $5 \mathrm{~b}$ are for $S=0$. Figs. 6 a and $6 \mathrm{~b}$ are similar results but for the $a=500$-nm particles. The refractive indices (water/polystyrene) used for different wavelengths in all calculations are determined by Eqs. (21) and (22), instead of being fixed.

Figs. 5 and 6 clearly show that the optical factors calculated by the T-matrix method are consistent with the experimental results of $R$ for different incident wavelengths, while the data calculated by RGD theory and Mie theory are quite divergent from the $R$ curve.

In Fig. 5, the measured $R$ changes from positive to negative values, while its values are always negative in Fig. 6. However, the optical factors calculated from the RGD theory (both coalescence and real doublet) are always positive in Figs. 5 and 6, which means that the RGD theory fails completely when the size parameter is large. The failure of RGD theory is foreseeable because the theory itself has been known to be valid only for small particles.

As $C_{2}$ (RGD) $/ C_{2}^{\prime}$ (RGD) in Eq. (2) is almost uniform for large size parameters, the curves for optical factors given by Eq. (2) are similar to Mie (coalescence) in shape. However, the results of optical factors of Mie (coalescence) and RGD (corrected) given by Eq. (2) are not proportional to the results of $R$. These errors may be caused by the assumption of coalescence, which does not really hold in the aggregate.

As shown by the experiments described above, among all different theories, the T-matrix method performs best in computing optical factors for large particles.

Our experiments also provide a useful clue as to how to improve the accuracy of the turbidity measurement. Since $R$ (the measurable quantity) or $F$ (the calculated quantity) changes with the incident wavelength $\lambda$, one should choose an appropriate wavelength to ensure that $R$ or $F$ is large enough; otherwise a tiny uncertainty of parameters in the calculation of $F$ may result in significant error. For example, at $\lambda=349 \mathrm{~nm}$ (in air), the refractive indices of water calculated by Eq. (21) in Figs. $2 \mathrm{a}$ and $2 \mathrm{~b}$ are 1.35357 and 1.34804 , respectively. So the difference of the refractive index caused by adding salinity is only 0.00553 or $0.4 \%$ of its value. With this tiny difference, the calculated optical factors in Figs. $2 \mathrm{a}$ and $2 \mathrm{~b}$ are -0.04582 and -0.05037 , respectively, or as high as $10 \%$ for its relative difference, although the absolute difference is only 0.00455 . On the other hand, with almost the same difference of the refractive indices of water, at $\lambda=785$ and $250 \mathrm{~nm}$, the relative differences in the calculated optical factors are only 0.5 and $1.3 \%$, respectively. In addition, when $R$ or $F$ is small, the relative measurement error also becomes large. Therefore, selecting the appropriate wavelength in the turbidity measurement to ensure that the change in turbidity is sensitive to the aggregation (having large $R$ ) is very crucial.

The optical factors calculated by the T-matrix method for a range of wavelengths and particle sizes are listed in Table 2. The salinity is $S=28$ and the temperature is $T=25^{\circ} \mathrm{C}$ in the calculation, and the refractive indices are calculated using Eqs. (21) and (22).

\section{Conclusions}

We have provided a detailed description of the calculation of optical factors using the T-matrix method. The necessity of correcting the refractive indices of particles and their surrounding medium for different incident wavelengths is discussed in particular. Apparently, an accurate calculation of the optical factor cannot be achieved without correct values of the refractive indices. Since the optical factor $F$ is proportional to a measurable quantity $R$, we were able to experimentally test the validity of all theories dealing with the calculation of $F$. Our experiments confirmed that the T-matrix method has obvious superiority over all other theories in providing a more accurate optical factor for dispersion of large particles. Therefore, our method is effective in extending the applicability range of the turbidity 
methodology and increasing measurement accuracy, as is concluded in our previous paper [2].

By careful discussion of the possible error in the calculation of optical factors and measurement of $R$ in this paper, we have also shown that the accuracy of turbidity measurement can be improved by choosing the appropriate wavelength to ensure that the turbidity is sensitive to the aggregation (having large $R$ or $F$ ). In addition, the data on the optical factors calculated by the T-matrix method for a range of particle radii and incident light wavelengths are provided.

\section{Acknowledgments}

This work is supported by Grants 10432060 and 10332050 from the National Natural Science Foundation of China and the "Knowledge Innovation Program" of the Chinese Academy of Sciences.

\section{References}

[1] J.W.Th. Lichtenbelt, H.J.M.C. Ras, P.H. Wiersema, J. Colloid Interface Sci. 46 (1974) 522

[2] Z.W. Sun, J. Liu, S.H. Xu, Langmuir 22 (2006) 4946.
[3] A.J. Cox, A.J. Deweerd, J. Linden, Am. J. Phys. 70 (2002) 620.

[4] H.C. van de Hulst, Light Scattering by Small Particles, Wiley, New York, 1957.

[5] M.I. Mishchenko, L.D. Travis, J. Quant. Spectrosc. Radiat. Transfer 60 (1998) 309

[6] J. Ding, L. Xu, J. Quant. Spectrosc. Radiat. Transfer 63 (1999) 163.

[7] M.I. Mishchenko, J. Opt. Soc. Am. A 8 (1991) 871.

[8] M.I. Mishchenko, Appl. Opt. 32 (1993) 4652.

[9] M.I. Mishchenko, L.D. Travis, A.A. Lacis, Scattering, Absorption, and Emission of Light by Small Particles, Cambridge Univ. Press, Cambridge, UK, 2002.

[10] M.I. Mishchenko, D.W. Mackowski, Opt. Lett. 19 (1994) 1604.

[11] A. Quirantes, A. Delgado, J. Quant. Spectrosc. Radiat. Transfer 78 (2003) 179.

[12] D.W. Mackowski, J. Opt. Soc. Am. A 11 (1994) 2851.

[13] A. Quirantes, F. Arroyo, J. Quirantes-Ros, J. Colloid Interface Sci. 240 (2001) 78.

[14] X. Quan, E.S. Fry, Appl. Opt. 34 (1995) 3477.

[15] G.T. McNeil, Opt. Eng. 16 (1977) 128

[16] W. Matthaus, Beitr. Meereskd. 33 (1974) 73

[17] X. Ma, J.Q. Lu, R.S. Brock, K.M. Jacobs, P. Yang, X.H. Hu, Phys. Med. Biol. 48 (2003) 4165.

[18] L.A. Matheson, J.L. Saunderson, in: R.H. Boundy, R.F. Boyer, S. Stoesser (Eds.), Styrene: Its Polymers, Copolymers, and Derivatives, Reinhold, New York, 1952.

[19] I.D. Nikolov, C.D. Ivanov, Appl. Opt. 39 (2000) 2067. 